\title{
Early response to adalimumab predicts long-term remission through 5 years of treatment in patients with ankylosing spondylitis
}

\author{
Joachim Sieper, ${ }^{1}$ Désirée van der Heijde, ${ }^{2}$ Maxime Dougados, ${ }^{3}$ L Steve Brown, ${ }^{4}$ \\ Frederic Lavie, ${ }^{5}$ Aileen L Pangan ${ }^{4}$
}

\begin{abstract}
'Department of
Gastroenterology, Infectiology and Rheumatology, Charité Universitätsmedizin Berlin, Berlin, Germany

2Department of Rheumatology, Leiden University Medical Center, Leiden, The Netherlands ${ }^{3}$ Rheumatology B Department, Paris-Descartes University, Cochin Hospital, Paris, France ${ }^{4}$ Abbott Laboratories, Abbott Park, Illinois, USA

${ }^{5}$ Abbott Laboratories, Rungis, France
\end{abstract}

\section{Correspondence to}

Joachim Sieper, University Clinic Benjamin Franklin, Medical Department I, Rheumatology, PO Box, Berlin 12200, Germany; joachim.sieper@charite.de

Received 24 June 2011 Accepted 9 October 2011 Published Online First 29 November 2011

\begin{abstract}
Objectives To describe the efficacy and safety through 5 years of adalimumab treatment in patients with ankylosing spondylitis (AS), and to identify predictors of remission.

Methods Patients with active AS were followed up to 5 years during a 24-week randomised, controlled period, followed by an open-label extension. Disease activity and clinical improvement were evaluated by Assessment in Spondyloarthritis International Society (ASAS) responses, Bath Ankylosing Spondylitis Disease Activity Index (BASDAl) and Ankylosing Spondylitis Disease Activity Score (ASDAS). Kaplan-Meier was used to identify patients with sustained ASAS partial remission (PR) or ASDAS inactive disease (ID) for three or more consecutive visits spanning $\geq 6$ months. Logistic regression was used to identify factors associated with remission. Explanatory variables included baseline demographic and disease characteristics and week 12 responses.
\end{abstract}

Results Of the 311 patients who received at least one dose of adalimumab, 202 (65\%) completed the 5-year study. Among 125 patients who received 5 years of adalimumab, 70\%, 77\%, 51\% and $61 \%$ achieved ASAS40, BASDAI 50, ASAS PR and ASDAS ID, respectively. Of 311 adalimumab-treated patients, $45 \%$ and $55 \%$ achieved sustained ASAS PR and ASDAS ID at any time during study participation. The strongest predictor of remission at years 1 and 5 and of sustained remission was achieving remission at 12 weeks of treatment; baseline characteristics showed weaker associations. Adverse events were comparable with previous reports on adalimumab safety.

Conclusions In patients with active AS, the efficacy and safety of adalimumab were maintained through 5 years with about half of the patients experiencing sustained remission at any time during the study. Early achievement of remission was the best predictor of long-term and sustained remission.

\section{INTRODUCTION}

Ankylosing spondylitis (AS) is a spondyloarthritis that presents with predominantly axial manifestations. Patients with AS may have inflammation of the spine, sacroiliac joints, peripheral joints and entheses.

Randomised controlled trials have consistently demonstrated the efficacy and safety of tumour necrosis factor (TNF) $\alpha$ inhibitors in reducing the signs and symptoms associated with AS.1-4 TNF inhibitors are the only class of drugs in addition to non-steroidal anti-inflammatory drugs (NSAIDs) proved to be effective for the axial component of AS, as reflected in the Assessment in Spondyloarthritis International Society (ASAS)/EULAR recommendations for the management of AS. ${ }^{5}$ Open-label extension studies support a sustained treatment effect for TNF inhibitors, ${ }^{6-9}$ but longer-term data are limited by small sample populations. ${ }^{10} 11$ The need for chronic disease management makes longterm data on effective treatments of clinical importance for both doctors and patients.

The ASAS consensus statement on the use of TNF inhibitors in axial spondyloarthritis recommends evaluating treatment response at least 12 weeks after initiation of TNF inhibitor treatment based on reductions in Bath Ankylosing Spondylitis Disease Activity Index (BASDAI) scores and the doctor's expert opinion to determine if TNF inhibitor use should continue. ${ }^{12}$ However, the implications of early treatment response on long-term disease control have not been fully explored. One report noted that a response at week 2 to infliximab, but not baseline disease characteristics, was associated with subsequent short-term clinical response. ${ }^{13}$ On the other hand, studies with infliximab, etanercept and/or adalimumab identified age, disease duration and baseline disease characteristics (raised C-reactive protein $(\mathrm{CRP})$, erythrocyte sedimentation rate, enthesitis, total back pain, BASDAI, Bath Ankylosing Spondylitis Functional Index (BASFI), human leucocyte antigen (HLA)-B27 positivity and lack of previous exposure to TNF inhibitors) as predictors of clinical response. ${ }^{14-20}$ These reports looked only at predictors of clinical response at 6 months or earlier and largely focused on improvement (ie, ASAS20, BASDAI 50).

The Adalimumab Trial evaluating Long-term efficacy and safety in AS (ATLAS) trial was a global, multicentre study of adalimumab in patients with active AS. ${ }^{1}$ This report summarises the clinical efficacy and safety of adalimumab through the end of the 5-year ATLAS trial and uses the efficacy data to determine predictors of long-term remission at years 1 and 5 and of sustained remission with adalimumab treatment.

\section{PATIENTS AND METHODS \\ Patients}

Patients were $\geq 18$ years of age, diagnosed with AS using the modified New York criteria, ${ }^{21}$ and had 
active disease based on the presence of at least two of the following three criteria: BASDAI score $\geq 4$, total back pain score $\geq 40 \mathrm{~mm}$ (on a 0-100 $\mathrm{mm}$ visual analogue scale (VAS)) and/or morning stiffness $\geq 1 \mathrm{~h}$. Patients must have had an inadequate response to, or intolerance of, NSAIDs. More detailed inclusion and exclusion criteria have been previously published. ${ }^{1}$

\section{Study design}

The ATLAS study was a phase 3, randomised, double-blind, placebo-controlled, multicentre trial conducted at 43 sites in the USA and Europe, designed to evaluate the safety and efficacy of adalimumab in patients with active AS (NCT00085644). ${ }^{1}$ Patients were randomised 2:1 to adalimumab (40 mg every other week) or placebo for 24 weeks of double-blind treatment, followed by the option to continue with open-label adalimumab by week 12 were eligible for early escape treatment with openlabel adalimumab. The study was conducted with the approval of the appropriate institutional ethics review board and with voluntary written informed patient consent.

\section{Clinical and safety measures}

Baseline demographics and disease characteristics were summarised at study entry. Efficacy and safety outcomes after 24 weeks and 2 years have been previously published. ${ }^{16}$ Efficacy assessments defined baseline as the last observation before the first adalimumab dose, either blinded or open-label. ASAS partial remission (ASAS PR) was defined as a value of $\leq 2$ (on a 0-10 scale) for all four domains: patient global assessment of disease activity, pain, function (BASFI) and inflammation (mean of BASDAI questions 5 and 6). ${ }^{22}$ The Ankylosing Spondylitis Disease Activity Score (ASDAS) was calculated from patient global assessment of disease activity (VAS), total back pain (BASDAI question 2), peripheral pain/swelling (BASDAI question 3), duration of morning stiffness (BASDAI question 6) and CRP (mg/l). ${ }^{23} 24$ ASDAS inactive disease (ASDAS ID) was defined as ASDAS <1.3, and major improvement in ASDAS (ASDAS MI) was defined as a decrease from baseline $\geq 2.0 .^{25}$ Treatment-emergent adverse events were those occurring on or after the first dose of adalimumab, up to 70 days after the last adalimumab dose. Adverse events are reported as number of events and as rate of events per 100 patient-years.

\section{Statistical analyses}

All patients who received at least one dose of blinded or openlabel adalimumab were included. Observed analyses were used to determine the proportion of patients achieving clinical responses after 1 and 5 years of adalimumab exposure and completers' analyses were used to determine the stability of response among those patients who completed the 5-year study. Only patients in the original double-blind adalimumab treatment group could have received 5 years of exposure.

\footnotetext{
Univariate regression analysis of long-term remission

Univariate logistic regression was used to evaluate factors hypothesised to be predictive of ASAS PR and ASDAS ID at 1 and 5 years of adalimumab exposure. Continuous explanatory variables included age, disease duration and the following baseline disease activity measures: BASFI, BASDAI, total back pain, patient and physician global assessments of disease activity and inflammation (mean of BASDAI questions 5 and 6). Colinearity
} in an extension period for up to a total of 5 years of study participation. Patients who failed to achieve an ASAS20 response

was assessed for continuous independent disease-state variables. Categorical independent variables included sex, presence of HLA-B27, abnormal baseline CRP $(\geq 0.49 \mathrm{mg} / \mathrm{dl})$, presence of baseline syndesmophytes and response after 12 weeks of adalimumab treatment as measured by the following outcome variables: ASAS20, ASAS40, ASAS PR, ASDAS ID, ASDAS MI and BASDAI 50.

\section{Multivariate regression analysis of sustained remission}

Kaplan-Meier survival curve analysis was used to determine the proportion of patients with sustained remission, defined as achieving ASAS PR or ASDAS ID on adalimumab treatment for at least three consecutive study visits spanning a period of at least 6 months at any point during the 5 -year study. Patients were censored if they withdrew prematurely from the trial. All variables considered in the univariate analysis for long-term remission were included in multivariate regression for sustained remission; additionally, the original treatment assignment was added as an explanatory variable to determine if achieving sustained remission differed based on initial randomisation. The analysis was performed for all patients with data available for all independent variables.

\section{RESULTS}

\section{Patient disposition and characteristics}

Data were collected from January 2004 through July 2009. Of the 315 patients enrolled, 208 patients were randomised to adalimumab and 107 to placebo. This analysis included 311 patients who received at least one dose of adalimumab during the double-blind or open-label portions of the trial ('any adalimumab exposure' set). This population was representative of patients with established AS: primarily male, white, HLA-B27-positive and with long disease duration (table 1).

Through 5 years, 113/315 (36\%) randomised patients discontinued the study, including 109/311 (35\%) patients with at least one dose of adalimumab. Investigators may have identified more than one reason for discontinuation, which was most commonly listed as due to adverse events, in $38 / 311(12 \%)$ patients and withdrawal of consent, in 37/311 (12\%) patients. The median duration of exposure to adalimumab among the 311 patients was 4.8 years (interquartile range $2.8-5.0$ years).

Table 1 Baseline demographics and disease characteristics

\begin{tabular}{|c|c|}
\hline Variable & Any ADA exposure $(\mathrm{N}=311)$ \\
\hline Sex, n (\%) male & $233(74.9)$ \\
\hline Race, n (\%) white & $299(96.1)$ \\
\hline Age, mean (SD) years & $42.3(11.6)$ \\
\hline HLA-B27+, n (\%) & $245(78.8)$ \\
\hline Disease duration, mean (SD) years & $11.00(9.5)$ \\
\hline Abnormal CRP, $\mathrm{n}(\%) \geq 0.49 \mathrm{mg} / \mathrm{dl}$ & $211(67.8)$ \\
\hline BASDAI, mean (SD) cm (0-10 VAS) & $6.3(1.7)$ \\
\hline $\begin{array}{l}\text { Patient global assessment, mean (SD) mm } \\
(0-100 \text { VAS })^{*}\end{array}$ & $63.6(20.9)$ \\
\hline $\begin{array}{l}\text { Physician global assessment, mean (SD) mm } \\
(0-100 \text { VAS })^{*}\end{array}$ & $57.3(18.6)$ \\
\hline Total back pain, mean (SD) mm (0-100 VAS) & $65.3(21.1)$ \\
\hline BASFI, mean (SD) mm (0-100 VAS) & $53.8(22.1)$ \\
\hline Inflammation, mean (SD) cm (0-10 VAS)† & $6.7(2.0)$ \\
\hline$\geq 1$ syndesmophyte, $\mathrm{n}(\%) \ddagger$ & $188(85.8)$ \\
\hline \multicolumn{2}{|c|}{$\begin{array}{l}{ }^{*} \mathrm{~N}=310 \text {. } \\
\text { †Mean of BASDAI question } 5 \text { and } 6 \text {. } \\
\ddagger \mathrm{N}=219 \text {. } \\
\text { ADA, adalimumab; BASDAI, Bath Ankylosing Spondylitis Disease Activity Index; BASFI, } \\
\text { Bath Ankylosing Spondylitis Functional Index; CRP, C-reactive protein; HLA, human } \\
\text { leucocyte antigen; VAS, visual analogue scale. }\end{array}$} \\
\hline
\end{tabular}




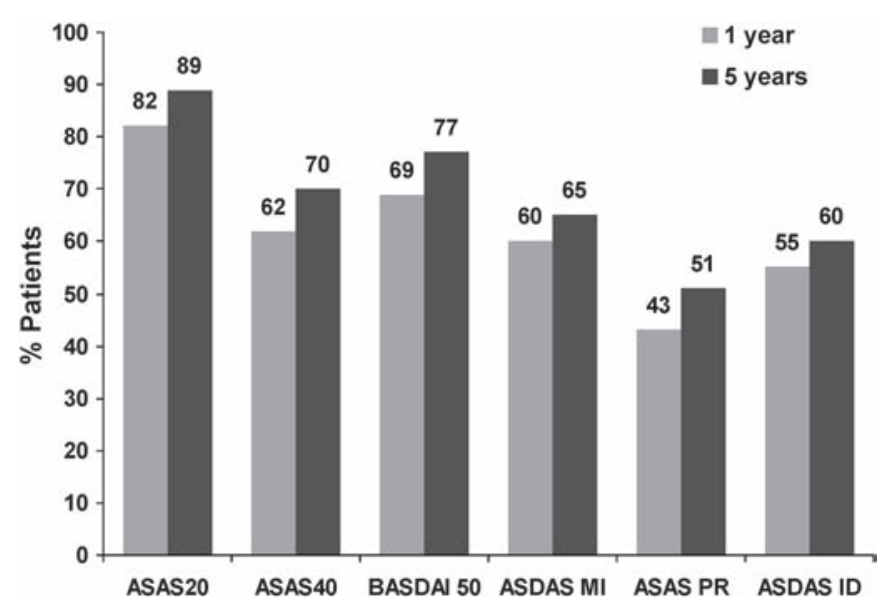

Figure 1 Percentages of patients achieving clinical response and remission states after 1 and 5 years of adalimumab treatment. Percentages based on patients originally randomised to adalimumab who completed the study and received 5 years of adalimumab $(\mathrm{N}=125)$. ASDAS MI: major improvement in ASDAS, decrease of $\geq 2.0$; ASAS PR: ASAS partial remission; ASDAS ID: inactive disease, ASDAS $<1.3$. ASAS, Assessment in Spondyloarthritis International Society; ASDAS, Ankylosing Spondylitis Disease Activity Score; BASDAl, Bath Ankylosing Spondylitis Disease Activity Index.

Table 2 Numbers and rates of adverse events of interest with tumour necrosis factor inhibitor treatment in patients with any adalimumab exposure ( $N=311,1201.1$ patient-years)

\begin{tabular}{lc}
\hline & N (E/100 PYs) \\
\hline Serious adverse events & $140(11.7)$ \\
Serious infections & $17(1.4)$ \\
Opportunistic infections* & $12(1.0)$ \\
Malignancies (excluding lymphoma and NMSC) & $3(0.2)$ \\
Lymphoma & $1(<0.1)$ \\
NMSC & $5(0.4)$ \\
Lupus-like syndrome & $1(<0.1)$ \\
Congestive heart failure-related & $2(0.2)$ \\
\hline
\end{tabular}

$\mathrm{E}$, events; NMSC, non-melanoma skin cancer; PYs, patient-years.

${ }^{*}$ All mild to moderate candidiasis

\section{Adalimumab efficacy through $\mathbf{5}$ years of treatment}

Improvements in the signs and symptoms of AS observed after 1 year were sustained through 5 years of treatment with adalimumab among those patients originally randomised to adalimumab who completed the study $(\mathrm{N}=125)$ (figure 1). Based on an observed analysis among patients with 1 or 5 years exposure, approximately half of the patients who had 1 year of adalimumab exposure achieved ASAS40 (143/282, 50.7\%), ASDAS MI (130/278, 46.8\%) and BASDAI 50 (169/282, 59.9\%) responses. At least one-third of patients experienced remission of disease activity after the first year of treatment (ASAS PR: 94/282, 33.3\%; ASDAS ID: 118/282, 41.8\%). The percentages of patients with good clinical responses were maintained among those originally randomised to adalimumab who completed 5 years of the study (ASAS40: 88/125, 70.4\%; ASDAS MI: 79/121, 65.3\%; BASDAI 50: 96/124, 77.4\%). Among patients who had 5 years of adalimumab exposure, the majority exhibited disease remission based on ASAS PR (63/124, 50.8\%) or ASDAS ID (75/124, 60.5\%) criteria.

\section{Long-term safety}

Over 1200 patient-years of adalimumab exposure were included in the long-term safety summary (table 2). There were no cases of tuberculosis or demyelinating disorder. Although there were no fatal adverse events, one non-treatment-emergent death occurred owing to adenocarcinoma at the gastro-oesophageal junction, with liver metastases $>1.2$ years after the patient discontinued adalimumab.

\section{Predictors of long-term and sustained remission}

Univariate analysis of long-term remission at 1 and 5 years of adalimumab treatment

Colinearity assessment of continuous explanatory baseline disease variables identified significant associations between all variables $(p<0.001)$. The highest colinearity was identified between total back pain, patient global assessment of disease activity, BASFI and BASDAI (correlation coefficients, $r=0.51-0.74$ ), while physician global assessment of disease activity and morning stiffness had an intermediate level of colinearity $(r=0.20-0.46)$.

Baseline characteristics including age, patient and physician global assessments of disease activity, total back pain, inflammation and BASFI generally showed significant negative associations with long-term remission at 1 and 5 years of adalimumab exposure; however, these effects were relatively small (figure 2). The presence of syndesmophytes at initiation of adalimumab treatment was noted to be a significant negative predictor of remission after 1 year of adalimumab treatment (OR (95\% CI), p value; ASAS PR: 0.29 (0.13 to 0.65), $p=0.003$; ASDAS ID: 0.35 (0.16- to 0.78$), p=0.01)$, but not of remission after 5 years of treatment.

The strongest predictors of ASAS PR or ASDAS ID after 1 and 5 years of adalimumab treatment were achievement of ASAS PR or ASDAS ID, respectively, at week 12 (figure 2). Patients who attained remission early were more than 10 times as likely to be in remission after 1 and 5 years of adalimumab treatment than those who did not achieve these disease states at week $12(p<0.001)$. Other measures of treatment response (ASAS20, ASAS40, ASDAS MI and BASDAI 50) after 12 weeks were also strongly predictive of long-term remission ( $O R$ ranged from approximately 3 to $9, \mathrm{p} \leq 0.01$ ).

\section{Multivariate analysis of sustained remission}

About half of the patients achieved a persistent state of disease remission. For ASAS PR, 141/311 (45\%) patients demonstrated sustained remission at any time during the study, while 170/311 $(55 \%)$ patients met the criteria for sustained ASDAS ID. The median time to event, including the 6 months of sustained response, was 364 days for both outcomes (figure 3 ). The proportion who achieved sustained remission and the median time to event were not appreciably different between the pool of patients with any adalimumab exposure $(\mathrm{N}=311)$ and the subgroup with all explanatory variable data available for the multivariate regression ( $N=207$, data not shown).

For both ASAS PR and ASDAS ID criteria, the respective achievement of these remission states at 12 weeks was the only significant positive predictor of sustained disease remission (figure 4). Reaching ASAS PR at week 12 resulted in a hazard ratio (95\% CI, p value) for sustained ASAS PR of 2.49 (1.40 to 4.43, $\mathrm{p}=0.002)$. Patients who reached ASDAS ID after 12 weeks of adalimumab had a hazard ratio $(95 \% \mathrm{CI}$, p value) of 3.18 (1.83 to 5.53, $\mathrm{p}<0.001$ ) for achieving sustained ASDAS ID. Additionally, decreased functional activity (higher BASFI) at baseline was significantly negatively associated with both sustained remission criteria, although the hazard ratios for both were close to 1.0 (ASAS PR: 0.98 (0.96 to 0.99), p=0.002; ASDAS ID: 0.99 (0.97 to 1.00$), p=0.04$ ). Indicators of baseline inflammation (abnormal 
A

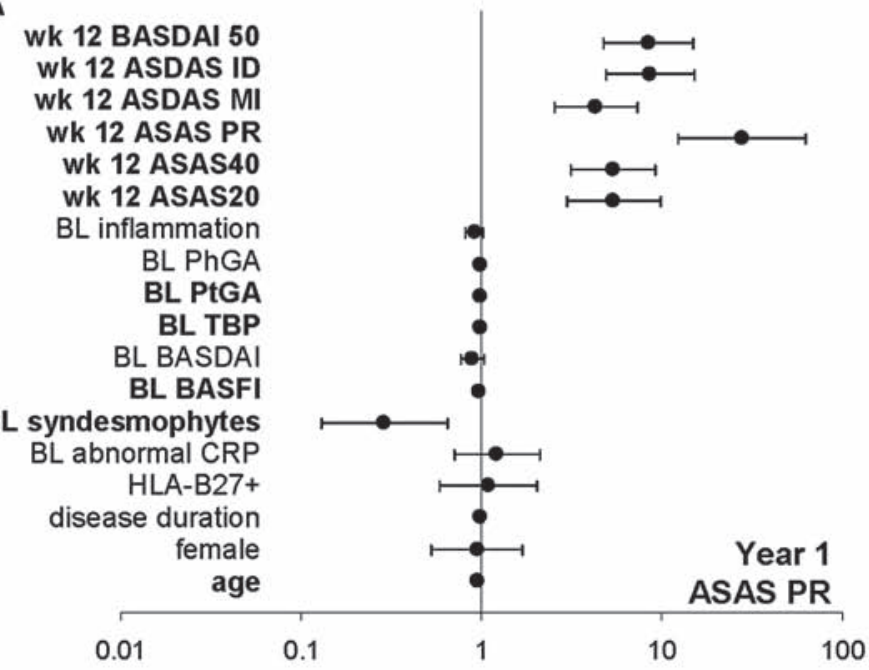

C

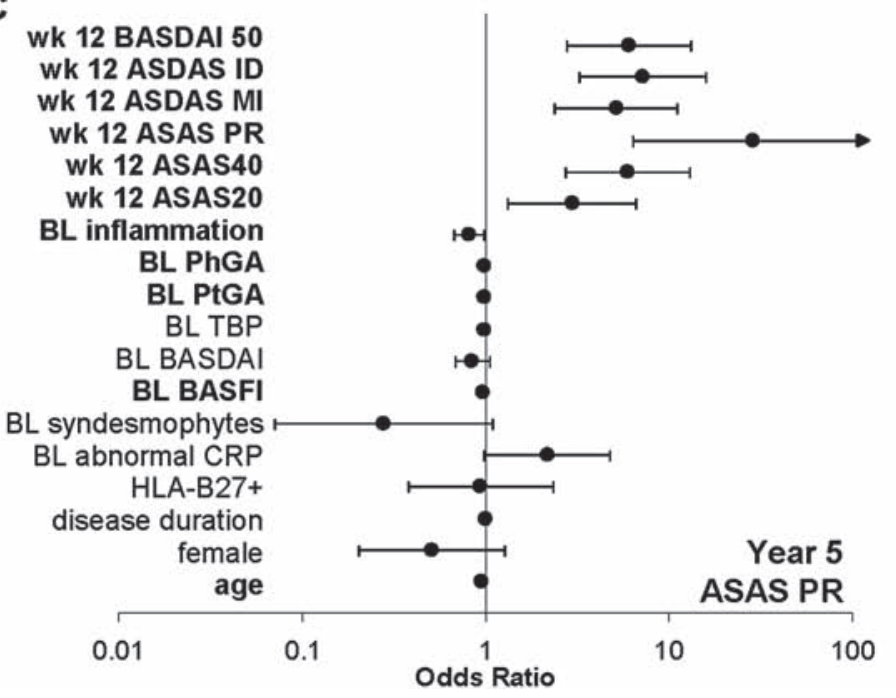

B

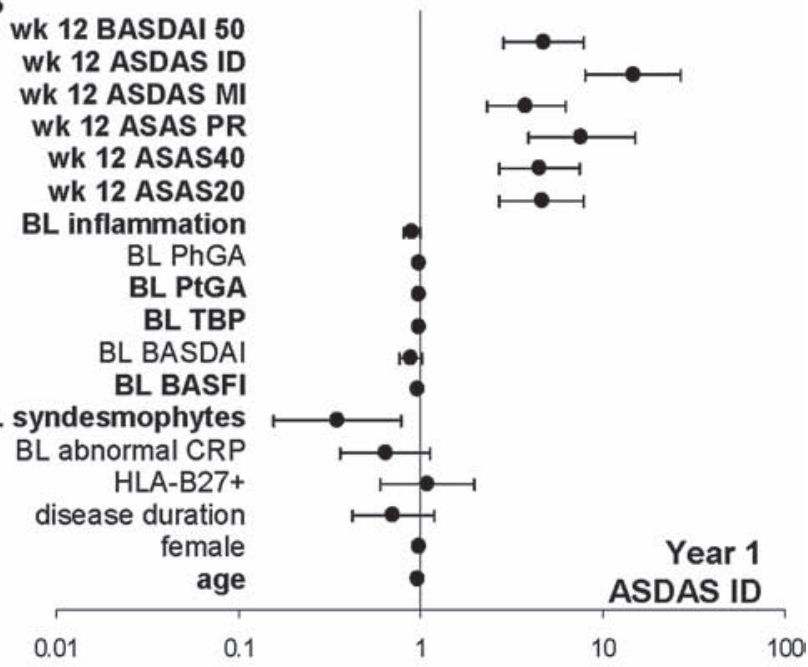

D

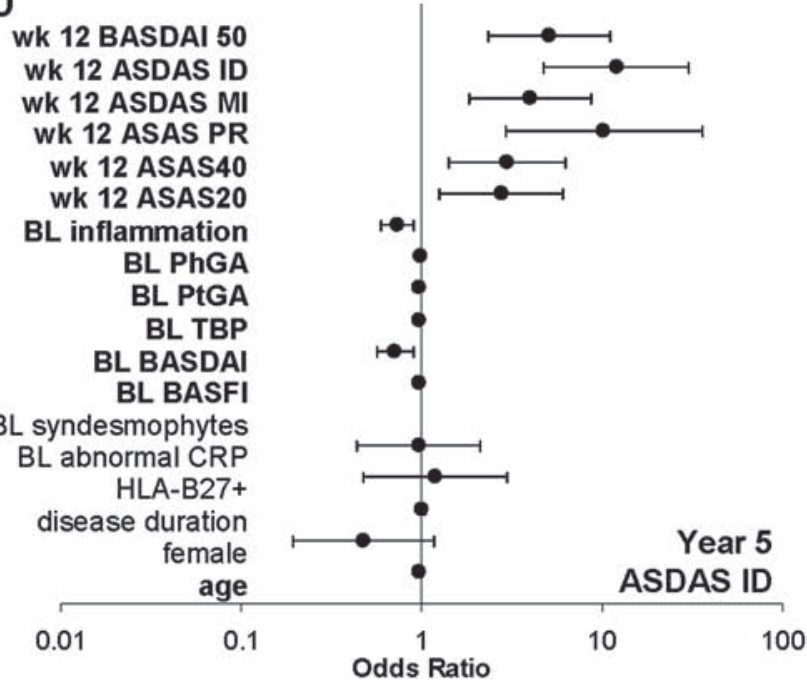

Figure 2 Odds ratio for achieving long-term remission after 1 or 5 years of adalimumab treatment by univariate logistic regression. (A) ASAS partial remission at 1 year; (B) ASDAS inactive disease at 1 year; (C) ASAS partial remission at 5 years; (D) ASDAS inactive disease at 5 years. Bold type indicates $p<0.05$. ASDAS ID: inactive disease, ASDAS <1.3; ASDAS MI: major improvement in ASDAS, decrease of $\geq 2.0$; ASAS PR: ASAS partial remission; BL: baseline; inflammation: mean of BASDAl questions 5 and 6; PhGA: physician global assessment of disease activity; PtGA: patient global assessment of disease activity; TBP: total back pain; abnormal C-reactive protein (CRP): $\geq 0.49 \mathrm{mg} / \mathrm{dl}$. ASAS, Assessment in Spondyloarthritis International Society; ASDAS, Ankylosing Spondylitis Disease Activity Score; BASDAI, Bath Ankylosing Spondylitis Disease Activity Index.

CRP: 0.62 (0.40 to 1.00), $\mathrm{p}=0.048$ and mean of BASDAI questions 5 and $6: 0.87$ ( 0.77 to 0.97$), p=0.015)$ were also significantly negatively associated with sustained ASDAS ID, but not sustained ASAS PR (figure 4).

\section{DISCUSSION}

The 5-year efficacy and safety of adalimumab treatment in patients with AS from the ATLAS trial are consistent with the reported observations through 2 years of exposure. ${ }^{6}$ Nearly two-thirds of the original patient population completed the 5 -year study. Among those who received 5 years of adalimumab treatment, the majority of patients demonstrated clinical response or benefit. Over half of the patients achieved remission based on criteria for ASAS PR (63/124, 50.8\%) or ASDAS ID $(75 / 124,60.5 \%)$. Similar long-term data from the open-label extension of a randomised controlled trial of etanercept in AS reported that $18 / 59$ patients $(30.5 \%)$ were in ASAS $\mathrm{PR}$ at 5 years. ${ }^{11}$ Infliximab use through 3 years showed that $15 / 46$ patients $(32.6 \%)$ demonstrated ASAS PR. ${ }^{26}$ The safety, the type and rate of adverse events seen through 5 years of adalimumab treatment were consistent with previous study reports and the known safety profile of adalimumab in other immune-mediated inflammatory diseases. ${ }^{1627}$ No new safety signals were noted.

Important therapeutic decisions are often made based on expected outcomes. Identifying factors that predict treatment response can assist clinicians in these management decisions. In a similar way, determining predictors of exceptional response, such as long-term disease remission over a long period, is helpful in shaping both the doctor's and the patient's expectations for chronic treatment. Previously published analyses predicting treatment response with anti-TNF treatment in patients with AS have examined short-term clinical responses ( $\leq 6$ months) and mainly focused on improvement, not disease remission. This is the first report in patients with AS of patterns and predictors of sustained remission, defined as ASAS PR or ASDAS ID for at least three consecutive study visits spanning a period of at least 6 months. 


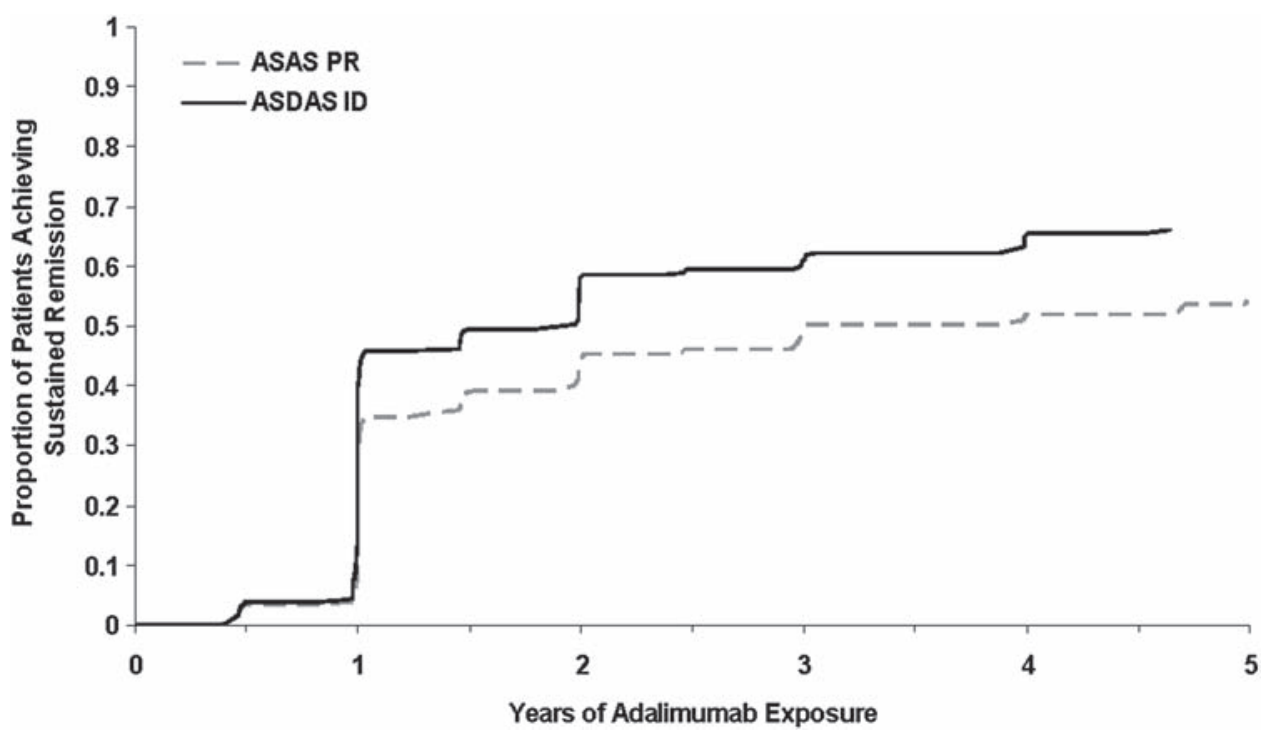

Figure 3 Kaplan-Meier analysis for achieving sustained remission. $\mathrm{N}=311$ patients who received at least 1 dose of adalimumab; patients who discontinued prematurely were not included at subsequent time points. Sustained remission was defined as ASAS partial remission (ASAS PR) or ASDAS inactive disease (ASDAS ID) for at least three consecutive study visits spanning at least 6 months. ASAS, Assessment in Spondyloarthritis International Society; ASDAS, Ankylosing Spondylitis Disease Activity Score.

Analyses aimed at predicting clinical outcomes have identified shorter disease duration and the closely related variable of younger patient age as relevant factors for treatment response at 3 or 6 months. ${ }^{15-17} 1920$ A randomised controlled trial of adalimumab in non-radiographic axial spondyloarthritis also suggested that younger age was associated with clinical outcomes after 1 year of treatment. ${ }^{28}$ Disease duration was not significantly associated with long-term remission in the univariate models described here, a discrepancy possibly due to the limited number of patients with short disease durations. This study does support the hypothesis that better outcomes are achieved in younger patients, as age was a significant negative predictor of long-term remission at 1 and 5 years of adalimumab treatment.

Baseline disease characteristics were often weakly predictive of long-term remission. Lower levels of baseline total back pain, inflammation, patient and physician global assessments of disease activity, BASDAI and BASFI increased the probability of a patient achieving remission at 1 and 5 years of adalimumab treatment. Randomised controlled trials, cohort and registry data have shown associations of baseline BASFI with achieving a BASDAI 50 response after 3-6 months of treatment. ${ }^{14-19}$ These same studies identified baseline CRP values as indicative of future response ${ }^{14-20}$; however, the focus was primarily on shortterm improvement and not long-term remission. An abnormal baseline CRP level was not a significant predictor of long-term remission as shown in the analyses conducted with the 5-year data from ATLAS. This indicates that raised CRP should not be a mandatory requirement for treatment of AS with TNF inhibitors. Patients with existing syndesmophytes at baseline were less likely to reach long-term remission in this study. Thus, initiation of anti-TNF treatment before the onset of new bone formation may enable a patient to achieve remission over the course of treatment.

In this study, the most notable factors that predicted longterm remission at years 1 and 5 of adalimumab treatment were early treatment responses at week 12, particularly achievement of remission. Most previous reports did not consider initial response as a potential indicator of future success. The hypothesis that short-term outcomes are more predictive of future response than baseline characteristics is supported by findings from an open-label infliximab study, which showed that an initial ASAS20 response at week 2 was predictive of ASAS20 at week 10; unfortunately, the impact of clinical improvement after 2 weeks of treatment was not evaluated relative to longerterm remission. ${ }^{13}$ Likewise, results presented here suggest that, more than the baseline disease characteristics, attainment of remission early in the course of treatment is predictive of longterm treatment success.

About half of the patients achieved sustained remission, the majority of whom met these criteria within a year of treatment initiation. In multivariate analyses, attainment of either ASAS PR or ASDAS ID at 12 weeks eliminated most baseline patient and disease characteristics from predictive models. A similar relationship between early treatment response and sustained remission has been described in a cohort of patients with rheumatoid arthritis. ${ }^{29}$ Although these results might initially appear to suggest that baseline variables have no impact on attaining sustained remission, the influence of baseline characteristics on attaining remission at 12 weeks must be considered.

These results are in alignment with current recommendations for evaluating treatment response after at least 12 weeks of treatment. ${ }^{12}{ }^{24}$ Achieving BASDAI 50 at week 12, in conjunction with expert opinion, has been identified as an appropriate gauge for continuation of anti-TNF treatment; results presented here suggest that patients who are in remission after 12 weeks of treatment (either by ASAS PR or ASDAS ID) are more likely to achieve long-term and sustained remission with continued adalimumab treatment.

One of the strengths of this analysis is the length of follow-up. It is also the first report to examine long-term remission by both ASAS PR and ASDAS ID criteria and the first to demonstrate early attainment of remission as a predictor of long-term and sustained remission. Limitations of this study include the openlabel, uncontrolled nature of the long-term post hoc analysis. There may be other characteristics related to sustained disease control that were not considered. The use of observed and completers' analyses may reflect a positive selection bias, since patients who remain in the study are likely to be those who 
A

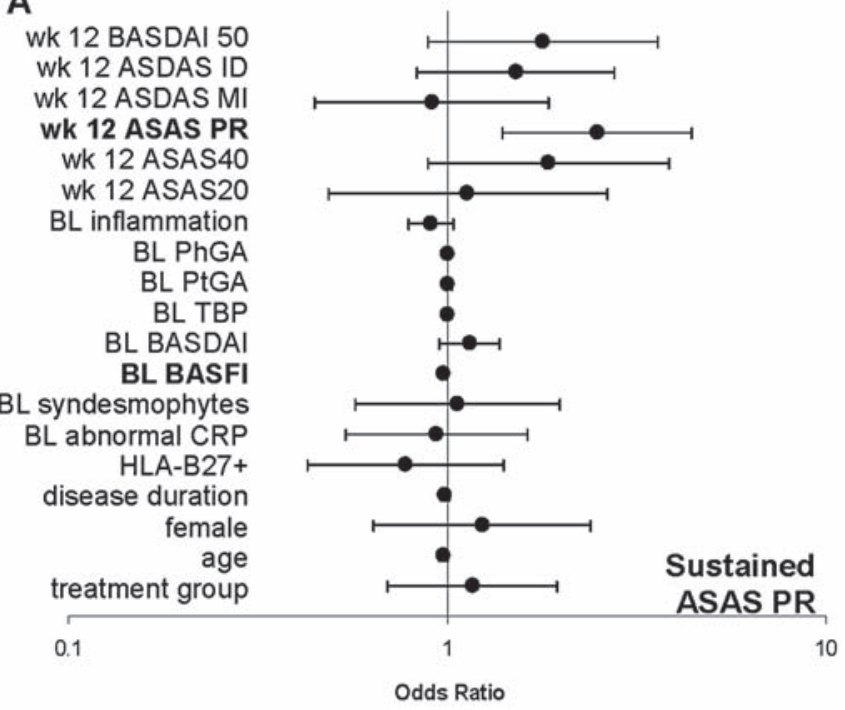

B

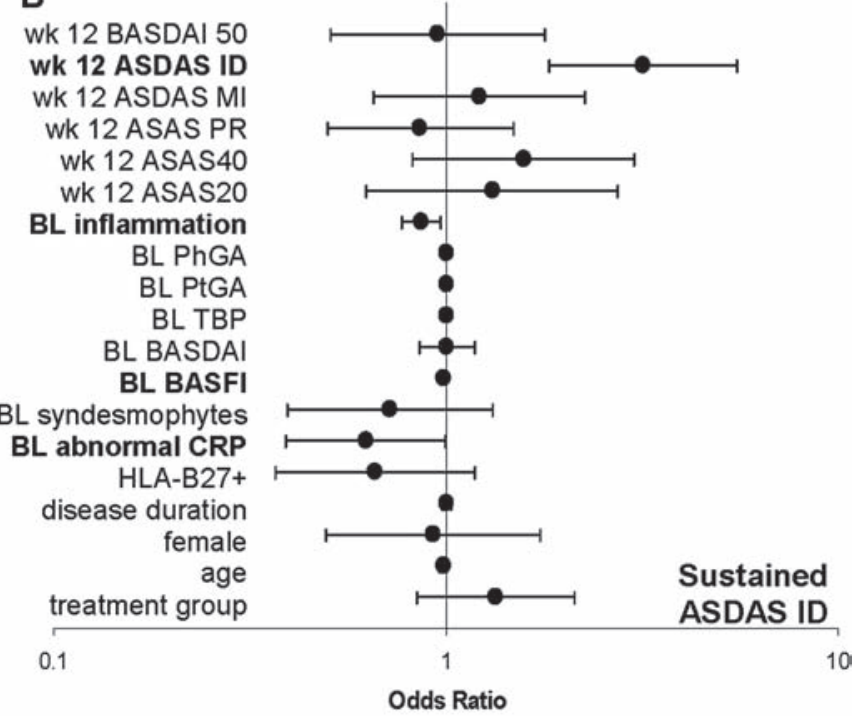

Figure 4 Odds ratio for achieving sustained remission by multivariate regression. (A) ASAS partial remission; (B) ASDAS inactive disease. Sustained remission was defined as ASAS partial remission or ASDAS inactive disease for at least three consecutive study visits spanning at least 6 months. Bold type indicates $p<0.05$. ASDAS ID: inactive disease, ASDAS <1.3; ASDAS MI: major improvement in ASDAS, decrease of $\geq 2.0$; ASAS PR: ASAS partial remission; $\mathrm{BL}$ : baseline; inflammation: mean of BASDAl questions 5 and 6; PhGA: physician global assessment of disease activity; PtGA: patient global assessment of disease activity; TBP: total back pain; abnormal C-reactive protein (CRP): $\geq 0.49 \mathrm{mg} / \mathrm{dl}$; treatment group: randomisation to either adalimumab or placebo during the first 24 weeks of the study. ASAS, Assessment in Spondyloarthritis International Society; ASDAS, Ankylosing Spondylitis Disease Activity Score; BASDAI, Bath Ankylosing Spondylitis Disease Activity Index.

benefit from the treatment and show good clinical response. Conversely, the possibility of a negative selection bias exists, as patients who discontinued owing to non-response early in the study might have achieved sustained remission with a longer duration of treatment.

The 5-year ATLAS data confirm that the early clinical improvements and safety profile seen with adalimumab in patients with AS are maintained through 5 years of treatment, supporting a favourable benefit:risk profile for the long-term use of adalimumab in patients with AS. Early achievement of remission with adalimumab was the most compelling predictor of subsequent long-term and sustained remission in patients with AS.

Funding Abbott Laboratories.

Competing interests JS has received research grants, speaker's fees, or consulting fees from Abbott, Merck and Pfizer. DvdH has received consulting fees from Abbott, Amgen, AstraZeneca, BMS, Centocor, Chugai, Eli-Lilly, GSK, Merck, Novartis, Otsuka, Pfizer, Roche, Sanofi-Aventis, Schering-Plough, UCB and Wyeth. MD has received research grants and consulting or speaker's fees from Abbott, BMS, Pfizer, Roche and UCB. LSB, FL and ALP are Abbott employees and may hold stock or options.

Provenance and peer review Not commissioned; externally peer reviewed.

Contributors All authors contributed to design, analysis and interpretation of these data, decided to publish the data and reviewed and approved the manuscript. Elaine $\mathrm{M} \mathrm{Smith}, \mathrm{PhD}$ of Abbott, drafted and revised the manuscript. ALP is the guarantor.

\section{REFERENCES}

1. van der Heijde D, Kivitz A, Schiff MH, et al. Efficacy and safety of adalimumab in patients with ankylosing spondylitis: results of a multicenter, randomized, doubleblind, placebo-controlled trial. Arthritis Rheum 2006;54:2136-46.

2. Davis JC Jr, Van Der Heijde D, Braun J, et al. Recombinant human tumor necrosis factor receptor (etanercept) for treating ankylosing spondylitis: a randomized, controlled trial. Arthritis Rheum 2003:48:3230-6.

3. van der Heijde D, Dijkmans B, Geusens P, et al. Efficacy and safety of infliximab in patients with ankylosing spondylitis: results of a randomized, placebo-controlled trial (ASSERT). Arthritis Rheum 2005;52:582-91.

4. Inman RD, Davis JC Jr, Heijde D, et al. Efficacy and safety of golimumab in patients with ankylosing spondylitis: results of a randomized, double-blind, placebo-controlled, phase III trial. Arthritis Rheum 2008;58:3402-12.

5. Braun J, van den Berg R, Baraliakos X, et al. 2010 update of the ASAS/EULAR recommendations for the management of ankylosing spondylitis. Ann Rheum Dis 2011;70:896-904.

6. van der Heijde D, Schiff MH, Sieper J, et al. Adalimumab effectiveness for the treatment of ankylosing spondylitis is maintained for up to 2 years: long-term results from the ATLAS trial. Ann Rheum Dis 2009;68:922-9.

7. Braun J, Deodhar A, Dijkmans B, et al. Efficacy and safety of infliximab in patients with ankylosing spondylitis over a two-year period. Arthritis Rheum 2008;59:1270-8.

8. Davis JC Jr, van der Heijde DM, Braun J, et al. Efficacy and safety of up to 192 weeks of etanercept therapy in patients with ankylosing spondylitis. Ann Rheum Dis 2008;67:346-52.

9. van der Heijde DM, Revicki DA, Gooch KL, et al. Physical function, disease activity and health-related quality-of-life outcomes after 3 years of adalimumab treatment in patients with ankylosing spondylitis. Arthritis Res Ther 2009;11:R124.

10. Braun J, Baraliakos X, Listing J, et al. Persistent clinical efficacy and safety of anti-tumour necrosis factor alpha therapy with infliximab in patients with ankylosing spondylitis over 5 years: evidence for different types of response. Ann Rheum Dis 2008;67:340-5.

11. Martín-Mola E, Sieper J, Leirisalo-Repo M, et al. Sustained efficacy and safety, including patient-reported outcomes, with etanercept treatment over 5 years in patients with ankylosing spondylitis. Clin Exp Rheumatol 2010;28:238-45.

12. van der Heijde D, Sieper J, Maksymowych WP, et al. 2010 Update of the international ASAS recommendations for the use of anti-TNF agents in patients with axial spondyloarthritis. Ann Rheum Dis 2011;70:905-8.

13. Huang $\mathbf{F}$, Zhu J, Zhang $L$, et al. Response to one infusion predicts subsequent improvement as well as the rate of relapse of ankylosing spondylitis infused with three pulses of infliximab. Clin Rheumatol 2007;26:920-6.

14. de Vries MK, van Eijk IC, van der Horst-Bruinsma IE, et al. Erythrocyte sedimentation rate, $\mathrm{C}$-reactive protein level and serum amyloid a protein for patient selection and monitoring of anti-tumor necrosis factor treatment in ankylosing spondylitis. Arthritis Rheum 2009;61:1484-90.

15. Rudwaleit M, Listing J, Brandt J, et al. Prediction of a major clinical response (BASDAI 50) to tumour necrosis factor alpha blockers in ankylosing spondylitis. Ann Rheum Dis 2004;63:665-70.

16. Glintborg B, Ostergaard M, Krogh NS, et al. Predictors of treatment response and drug continuation in 842 patients with ankylosing spondylitis treated with anti-tumour necrosis factor: results from 8 years' surveillance in the Danish nationwide DANBIO registry. Ann Rheum Dis 2010;69:2002-8.

17. Rudwaleit M, Claudepierre P, Wordsworth $\mathrm{P}$, et al. Effectiveness, safety and predictors of good clinical response in 1250 patients treated with adalimumab for active ankylosing spondylitis. J Rheumatol 2009;36:801-8.

18. Lord PA, Farragher TM, Lunt M, et al. Predictors of response to anti-TNF therapy in ankylosing spondylitis: results from the British Society for Rheumatology Biologics Register. Rheumatology (Oxford) 2010;49:563-70.

19. Vastesaeger $\mathbf{N}$, van der Heijde D, Inman RD, et al. Predicting the outcome of ankylosing spondylitis therapy. Ann Rheum Dis 2011;70:973-81. 
20. Arends S, Brouwer $\mathrm{E}$, van der Veer $\mathrm{E}$, et al. Baseline predictors of response and discontinuation of tumor necrosis factor-alpha blocking therapy in ankylosing spondylitis: a prospective longitudinal observational cohort study. Arthritis Res Ther 2011;13:94.

21. van der Linden $\mathbf{S}$, Valkenburg HA, Cats A. Evaluation of diagnostic criteria for ankylosing spondylitis. A proposal for modification of the New York criteria. Arthritis Rheum 1984;27:361-8.

22. Anderson JJ, Baron G, van der Heijde D, et al. Ankylosing spondylitis assessment group preliminary definition of short-term improvement in ankylosing spondylitis. Arthritis Rheum 2001;44:1876-86.

23. van der Heijde D, Lie E, Kvien TK, et al. ASDAS, a highly discriminatory ASASendorsed disease activity score in patients with ankylosing spondylitis. Ann Rheum Dis 2009:68:1811-8

24. Sieper J, Rudwaleit M, Baraliakos X, et al. The assessment of spondyloarthritis international society (asas) handbook: a guide to assess spondyloarthritis. Ann Rheum Dis 2009;68:1-44.
25. Machado P, Landewé R, Lie E, et al. Ankylosing Spondylitis Disease Activity Score (ASDAS): defining cut-off values for disease activity states and improvement scores. Ann Rheum Dis 2011;70:47-53.

26. Braun J, Baraliakos X, Brandt J, et al. Persistent clinical response to the antiTNF-alpha antibody infliximab in patients with ankylosing spondylitis over 3 years. Rheumatology (Oxford) 2005;44:670-6.

27. Burmester GR, Mease P, Dijkmans BA, et al. Adalimumab safety and mortality rates from global clinical trials of six immune-mediated inflammatory diseases. Ann Rheum Dis 2009;68:1863-9.

28. Haibel $\mathbf{H}$, Rudwaleit $\mathrm{M}$, Listing $\mathrm{J}$, et al. Efficacy of adalimumab in the treatment of axial spondylarthritis without radiographically defined sacroiliitis: results of a twelveweek randomized, double-blind, placebo-controlled trial followed by an open-label extension up to week fifty-two. Arthritis Rheum 2008;58:1981-91.

29. Schipper LG, Fransen J, den Broeder AA, et al. Time to achieve remission determines time to be in remission. Arthritis Res Ther 2010;12:R97. 